\title{
Strong Ergodic Theorems for Non-Lipschitzian Mappings of Asymptotically Nonexpansive Type in Uniformly Convex Banach Spaces
}

\author{
Isao MIYADERA \\ Waseda University
}

\begin{abstract}
In this paper we establish strong ergodic theorems for non-Lipschitzian mappings of asymptotically nonexpansive type in uniformly convex Banach spaces.
\end{abstract}

\section{Introduction.}

Throughout this paper $X$ denotes a uniformly convex Banach space, $C$ a nonempty bounded closed convex subset of $X$, and $T$ a mapping from $C$ into itself.

The asymptotic behavior of asymptotically nonexpansive mappings has been studied by many authors. There appear in the literature the following three definitions of an asymptotically nonexpansive mapping:

(c ${ }_{1}$ (Goebel and Kirk [3]) There exists a sequence $\left\{a_{k}\right\}$ with $\lim _{k \rightarrow \infty} a_{k}=1$ such that $\left\|T^{k} u-T^{k} v\right\| \leqslant a_{k}\|u-v\|$ for $u, v \in C$ and integers $k \geqslant 0$. In this case we say that $T$ is asymptotically nonexpansive in the strong sense.

$\left(c_{2}\right) \quad\left(\right.$ Kirk [4]) $T^{K}$ is continuous for some positive integer $K$ and

$$
\overline{\lim }_{k \rightarrow \infty} \sup _{v \in C}\left(\left\|T^{k} u-T^{k} v\right\|-\|u-v\|\right) \leqslant 0 \quad \text { for } \quad u \in C .
$$

In this case we say that $T$ is asymptotically nonexpansive in the weak sense.

$\left(c_{3}\right)$ (Bruck, Kuczumow and Reich [2]) $T$ is called asymptotically nonexpansive in the intermediate sense if $T^{K}$ is continuous for some positive integer $K$ and

$$
\varlimsup_{k \rightarrow \infty} \sup _{u, v \in C}\left(\left\|T^{k} u-T^{k} v\right\|-\|u-v\|\right) \leqslant 0 \text {. }
$$

Definition 0.1. A sequence $\left\{x_{n}\right\}_{n \geqslant 0}$ in $X$ is said to be strongly almost convergent to an element $x$ in $X$ if (the strong limit) $\lim _{n \rightarrow \infty}(1 / n) \sum_{i=0}^{n-1} x_{i+k}=x$ uniformly in $k=0,1,2, \cdots$.

The purpose of this paper is to prove the following strong ergodic theorems.

Received July 10, 1997 
THEOREM 0.1. Let $T$ be asymptotically nonexpansive in the weak sense, and let $x$ be an element in $C$. The following (a) and (b) are equivalent:

(a) $\left\{T^{n} x\right\}$ is strongly almost convergent to a fixed point of $T$.

(b) $\lim _{l, m, n \rightarrow \infty}\left\|\frac{1}{2}\left(S_{n} T^{l+n} x+S_{m} T^{l+m} x\right)-T^{l}\left(\frac{1}{2} S_{n} T^{n} x+\frac{1}{2} S_{m} T^{m} x\right)\right\|=0$, where $S_{n}=(1 / n) \sum_{i=0}^{n-1} T^{i}$ for $n=1,2, \cdots$.

THEOREM 0.2. Let T be asymptotically nonexpansive in the intermediate sense, and let $x$ be an element in $C$. If

$$
\lim _{n \rightarrow \infty}\left\|T^{n+i} x-T^{n} x\right\| \text { exists uniformly in } i=1,2, \cdots,
$$

then $\left\{T^{n} x\right\}$ is strongly almost convergent to a fixed point of $T$.

REMARKS 0.1 . 1) Since $\lim _{n \rightarrow \infty}\left\|T^{n+i} x-T^{n} x\right\|$ exists for every $i=1,2, \cdots$, in (0.3) we require the uniformity of the limit in $i$. We can prove that if there exists a subsequence $\left\{n_{k}\right\}$ of $\{n\}$ such that

$$
\lim _{k \rightarrow \infty}\left\|T^{n_{k}+i} x-T^{n_{k}} x\right\| \text { exists uniformly in } i=1,2, \cdots
$$

then (0.3) is satisfied. We see also that if $\left\{T^{n_{k}} x\right\}$ is strongly convergent for some subsequence $\left\{n_{k}\right\}$ of $\{n\}$ then (0.4) and hence (0.3) is satisfied. So, in particular, if $T$ is compact then (0.3) is satisfied. 2) Clearly if $T$ is asymptotically nonexpansive in the strong sense then it is asymptotically nonexpansive in the intermediate sense. But the converse does not hold (see Example 0.1 below). So Theorem 0.2 is a generalization of the result by Oka [7] and Krüppel and Górnicki [6]. 3) Theorems 0.1 and 0.2 can be generalized to the case of almost-orbits of $T$ (see $\S 2$ ).

Example 0.1 . Let $C=[0,1]$ and $\varphi$ be the Cantor ternary function. We define $T: C \rightarrow C$ by

$$
\begin{aligned}
& T u=u / 2 \quad \text { if } \quad 0 \leqslant u \leqslant 1 / 2 \text {, } \\
& =\varphi(u) / 2 \text { if } \quad 1 / 2<u \leqslant 1 .
\end{aligned}
$$

Then for $n=1,2, \cdots, T^{n} u=u / 2^{n}(0 \leqslant u \leqslant 1 / 2)$ and $T^{n} u=\varphi(u) / 2^{n}(1 / 2<u \leqslant 1)$. Therefore, for each $n \geqslant 1, T^{n}$ is continuous, but it is not Lipschitz continuous and a fortiori $T$ is not asymptotically nonexpansive in the strong sense. Since $\sup _{u, v \in[0,1]}\left(\left|T^{n} u-T^{n} v\right|-\right.$ $|u-v|) \leqslant 1 / 2^{n-1} \rightarrow 0$ as $n \rightarrow \infty, T$ is asymptotically nonexpansive in the intermediate sense.

\section{Proofs of theorems.}

We see that for every sequence $\left\{x_{n}\right\}_{n} \geqslant 0$ in $X$ the following equality holds true: For any $l, p \geqslant 1$ and $k \geqslant 0$ 


$$
\frac{1}{l} \sum_{i=0}^{l-1} x_{i+k}=\frac{1}{l} \sum_{i=0}^{l-1}\left(\frac{1}{p} \sum_{j=0}^{p-1} x_{i+j+k}\right)+\frac{1}{l p} \sum_{i=1}^{p-1}(p-i)\left(x_{i+k-1}-x_{i+k+l-1}\right) .
$$

Similarly as in the proof of [5, Lemma 2] we have

LEMMA 1.1. Let $T$ be asymptotically nonexpansive in the weak sense and let $x$ be an element in $C$. If (b) in Theorem 0.1 is satisfied, then $\left\{\left\|S_{n} T^{n} x-f\right\|\right\}$ is convergent for every $f \in F(T)$, where $F(T)$ denotes the set of fixed points of $T$.

REMARK 1.1. We note that $F(T)$ is not empty if $T$ is asymptotically nonexpansive in the weak sense. See [4].

LEMMA 1.2. Let $T$ be asymptotically nonexpansive in the weak sense and let $x$ be an element in $C$. If (b) in Theorem 0.1 is satisfied, then $\left\{S_{n} T^{n} x\right\}$ is strongly convergent to a fixed point of $T$.

Proof. Take an $f \in F(T)$ and set $u_{n}=S_{n} T^{n} x-f$ for $n \geqslant 1$. By Lemma $1.1,\left\{\left\|u_{n}\right\|\right\}$ is convergent. Set $d=\lim _{n \rightarrow \infty}\left\|u_{n}\right\|$. By $\lim _{n \rightarrow \infty}\left\|u_{n+1}-u_{n}\right\|=0$ we have

$$
\lim _{n \rightarrow \infty}\left\|u_{n+i}+u_{n}\right\|=2 d \quad \text { for every } i \geqslant 0 \text {. }
$$

Since $S_{n+k} T^{n+k} x=(n+k)^{-1} \sum_{i=0}^{n+k-1} S_{n} T^{n+k+i} x+v(n, k)$ by $(1.1)$ and $\|v(n, k)\| \leqslant(n-$ 1) $\operatorname{diam} C / 2(n+k)$, where $v(n, k)=[n(n+k)]^{-1} \sum_{i=1}^{n-1}(n-i)\left[T^{n+k+i-1} x-T^{2(n+k)+i-1} x\right]$ and diam $C$ denotes the diameter of $C$, we get

$$
\begin{aligned}
\left\|u_{n+k}+u_{m+k}\right\|= & \| \frac{1}{n+k} \sum_{i=0}^{n+k+1}\left(S_{n} T^{n+k+i} x+S_{m} T^{m+k+i} x-2 f\right) \\
& +\frac{n-m}{(m+k)(n+k)} \sum_{i=0}^{n+k-1}\left(S_{m} T^{m+k+i} x-f\right)+v(n, k)+v(m, k) \\
& +\frac{1}{m+k} \sum_{i=n+k}^{m+k-1}\left(S_{m} T^{m+k+i} x-f\right) \| \\
\leq & \frac{2}{n+k} \sum_{i=0}^{n+k-1}\left\|\frac{1}{2}\left(S_{n} T^{n+k+i} x+S_{m} T^{m+k+i} x\right)-f\right\| \\
& +\left[\frac{2(m-n)}{m+k}+\frac{n-1}{2(n+k)}+\frac{m-1}{2(m+k)}\right] \operatorname{diam} C
\end{aligned}
$$

for $m \geqslant n \geqslant 1$ and $k \geqslant 0$. By (b) and (0.1), for any $\varepsilon>0$ there exists an integer $N(\varepsilon)>0$ such that

$$
\begin{gathered}
\left\|\frac{1}{2}\left(S_{n} T^{l+n} x+S_{m} T^{l+m} x\right)-T^{l}\left(S_{n} T^{n} x / 2+S_{m} T^{m} x / 2\right)\right\|<\varepsilon / 2, \\
\left\|T^{l}\left(S_{n} T^{n} x / 2+S_{m} T^{m} x / 2\right)-f\right\|<\varepsilon / 2+\left\|S_{n} T^{n} x / 2+S_{m} T^{m} x / 2-f\right\|
\end{gathered}
$$

for $l, m, n \geqslant N(\varepsilon)$. Therefore, if $m \geqslant n \geqslant N(\varepsilon)$ and $k \geqslant N(\varepsilon)$ then 


$$
\begin{aligned}
& \left\|\left(S_{n} T^{n+k+i} x+S_{m} T^{m+k+i} x\right) / 2-f\right\| \\
& \quad \leqslant \quad\left\|\left(S_{n} T^{n+k+i} x+S_{m} T^{m+k+i} x\right) / 2-T^{k+i}\left(S_{n} T^{n} x / 2+S_{m} T^{m} x / 2\right)\right\| \\
& \quad+\left\|T^{k+i}\left(S_{n} T^{n} x / 2+S_{m} T^{m} x / 2\right)-f\right\|<\varepsilon / 2+\varepsilon / 2+\left\|S_{n} T^{n} x / 2+S_{m} T^{m} x / 2-f\right\| \\
& \quad \leqslant \varepsilon+\left\|u_{n}+u_{m}\right\| / 2
\end{aligned}
$$

for every $i \geqslant 0$. Combining this with (1.3) we obtain

$$
\left\|u_{n+k}+u_{m+k}\right\| \leqslant 2 \varepsilon+\left\|u_{n}+u_{m}\right\|+\left[\frac{2(m-n)}{m+k}+\frac{n-1}{2(n+k)}+\frac{m-1}{2(m+k)}\right] \operatorname{diam} C
$$

for $m \geqslant n \geqslant N(\varepsilon)$ and $k \geqslant N(\varepsilon)$. Letting $k \rightarrow \infty$, we see from (1.2) that

$$
2 d \leqslant 2 \varepsilon+\left\|u_{n}+u_{m}\right\| \leqslant 2 \varepsilon+\left\|u_{n}\right\|+\left\|u_{m}\right\| \quad \text { for every } m, n \geqslant N(\varepsilon) .
$$

This shows that $\lim _{n, m \rightarrow \infty}\left\|u_{n}+u_{m}\right\|=2 d$. Since $\left\|u_{n}\right\| \rightarrow d$ as $n \rightarrow \infty$, the uniform convexity of $X$ implies $\lim _{n, m \rightarrow \infty}\left\|S_{n} T^{n} x-S_{m} T^{m} x\right\|=\lim _{n, m \rightarrow \infty}\left\|u_{n}-u_{m}\right\|=0$, whence $\left\{S_{n} T^{n} x\right\}$ converges strongly. Put $y=\lim _{n \rightarrow \infty} S_{n} T^{n} x$.

We want to show $y \in F(T)$. Since for $l \geqslant 0,\left\|S_{n} T^{l+n} x-S_{n} T^{n} x\right\| \rightarrow 0$ as $n \rightarrow \infty$, we have

$$
\lim _{n \rightarrow \infty} S_{n} T^{l+n} x=y \quad \text { for every } l \geqslant 0 .
$$

Let $\varepsilon>0$ be arbitrarily given. By (b) with $m=n$ and (0.1) there exists an integer $N_{1}(\varepsilon)>0$ such that $\left\|T^{l} S_{n} T^{n} x-S_{n} T^{l+n} x\right\|<\varepsilon$ and $\left\|T^{l} S_{n} T^{n} x-T^{l} y\right\|<\varepsilon+\left\|S_{n} T^{n} x-y\right\|$ for $l, n \geqslant N_{1}(\varepsilon)$, which implies

$$
\begin{aligned}
\left\|T^{l} y-y\right\| & \leqslant\left\|T^{l} y-T^{l} S_{n} T^{n} x\right\|+\left\|T^{l} S_{n} T^{n} x-S_{n} T^{l+n} x\right\|+\left\|S_{n} T^{l+n} x-y\right\| \\
& <2 \varepsilon+\left\|S_{n} T^{n} x-y\right\|+\left\|S_{n} T^{l+n} x-y\right\| \quad \text { for } l, n \geqslant N_{1}(\varepsilon) .
\end{aligned}
$$

Letting $n \rightarrow \infty$, it follows from (1.4) that $\left\|T^{l} y-y\right\| \leqslant 2 \varepsilon$ for $l \geqslant N_{1}(\varepsilon)$. Therefore we obtain $\lim _{l \rightarrow \infty} T^{l} y=y$. Combining this with the continuity of $T^{K}$ we have $y \in F(T)$. Q.E.D.

Proof of Theorem 0.1. Suppose that there exists an element $y \in F(T)$ such that $\lim _{n \rightarrow \infty} S_{n} T^{k} x=y$ uniformly in $k \geqslant 0$. By this assumption and $(0.1)$, for any $\varepsilon>0$ there exists an integer $N(\varepsilon)>0$ such that if $n, l \geqslant N(\varepsilon)$ then $\left\|S_{n} T^{k} x-y\right\|<\varepsilon / 3$ for $k \geqslant 0$ and $\left\|T^{l} u-y\right\|<\|u-y\|+\varepsilon / 3$ for $u \in C$. Therefore, if $l, m, n \geqslant N(\varepsilon)$ then we have

$$
\begin{aligned}
& \left\|\frac{1}{2}\left(S_{n} T^{l+n} x+S_{m} T^{l+m} x\right)-T^{l}\left(\frac{1}{2} S_{n} T^{n} x+\frac{1}{2} S_{m} T^{m} x\right)\right\| \\
\leqslant & \left\|\frac{1}{2}\left(S_{n} T^{l+n} x+S_{m} T^{l+m} x\right)-y\right\|+\left\|T^{l}\left(\frac{1}{2} S_{n} T^{n} x+\frac{1}{2} S_{m} T^{m} x\right)-y\right\| \\
< & \frac{1}{2}\left(\left\|S_{n} T^{l+n} x-y\right\|+\left\|S_{m} T^{l+m} x-y\right\|\right)+\left\|\frac{1}{2}\left(S_{n} T^{n} x+S_{m} T^{m} x\right)-y\right\|+\varepsilon / 3<\varepsilon .
\end{aligned}
$$

So, (b) holds good.

Conversely, suppose that (b) is satisfied. By virtue of Lemma 1.2 there exists an element $y \in F(T)$ such that $\lim _{n \rightarrow \infty} S_{n} T^{n} x=y$. This implies 


$$
\lim _{n, l \rightarrow \infty} S_{n} T^{n+l+k} x=y \quad \text { uniformly in } k \geqslant 0 .
$$

In fact, let $\varepsilon>0$ be arbitrarily given. By (b) with $m=n$, there exists an integer $N_{1}(\varepsilon)>0$ such that $\left\|S_{n} T^{n+j} x-T^{j} S_{n} T^{n} x\right\|<\varepsilon / 3$ for $j, n \geqslant N_{1}(\varepsilon)$. So, if $l, n \geqslant N_{1}(\varepsilon)$ then $\| S_{n} T^{n+l+k} x-$ $T^{l+k} S_{n} T^{n} x \|<\varepsilon / 3$ for every $k \geqslant 0$. By $(0.1)$ and $\lim _{n \rightarrow \infty} S_{n} T^{n} x=y$, there exists an integer $N_{2}(\varepsilon)>0$ such that if $l, n \geqslant N_{2}(\varepsilon)$ then $\left\|T^{l+k} S_{n} T^{n} x-y\right\|<\varepsilon / 3+\left\|S_{n} T^{n} x-y\right\|<2 \varepsilon / 3$ for every $k \geqslant 0$. Consequently, if $n, l \geqslant \max \left\{N_{1}(\varepsilon), N_{2}(\varepsilon)\right\}$ then $\left\|S_{n} T^{n+l+k} x-y\right\| \leqslant$ $\left\|S_{n} T^{n+l+k} x-T^{l+k} S_{n} T^{n} x\right\|+\left\|T^{l+k} S_{n} T^{n} x-y\right\|<\varepsilon$ for every $k \geqslant 0$. So we have (1.5).

By (1.5), for any $\varepsilon>0$ there exists an integer $N(=N(\varepsilon))>0$ such that

$$
\left\|S_{N} T^{2 N+k} x-y\right\|<\varepsilon \quad \text { for every } k \geqslant 0 .
$$

Since $S_{n} T^{k} x=(1 / n) \sum_{i=0}^{2 N-1} S_{N} T^{k+i} x+(1 / n) \sum_{i=0}^{n-2 N-1} S_{N} T^{2 N+k+i} x+(1 / n N) \sum_{i=1}^{N-1}(N-i)$ $\times\left(T^{k+i-1} x-T^{k+i+n-1} x\right)$ by (1.1), we see from (1.6) that if $n>2 N$ then

$$
\begin{aligned}
\left\|S_{n} T^{k} x-y\right\| \leqslant & \left\|\frac{1}{n} \sum_{i=0}^{2 N-1}\left(S_{N} T^{k+i} x-y\right)\right\|+\left\|\frac{1}{n} \sum_{i=0}^{n-2 N-1}\left(S_{N} T^{2 N+k+i} x-y\right)\right\| \\
& +\frac{N-1}{2 n} \operatorname{diam} C \leq \frac{2 N}{n} \operatorname{diam} C+\frac{1}{n} \sum_{i=0}^{n-2 N-1}\left\|S_{N} T^{2 N+k+i} x-y\right\| \\
& +\frac{N-1}{2 n} \operatorname{diam} C \\
< & \left(\frac{2 N}{n}+\frac{N-1}{2 n}\right) \operatorname{diam} C+\varepsilon \quad \text { for every } k \geqslant 0 .
\end{aligned}
$$

Therefore $\varlimsup_{n \rightarrow \infty} \sup _{k \geqslant 0}\left\|S_{n} T^{k} x-y\right\| \leqslant \varepsilon$, i.e., $\lim _{n \rightarrow \infty} \sup _{k \geqslant 0}\left\|S_{n} T^{k} x-y\right\|=0$. Q.E.D.

Proof of Theorem 0.2. Suppose that $(0.3)$ is satisfied. It suffices to show that (b) in Theorem 0.1 is satisfied. Let $\varepsilon>0$ be arbitrarily given. By virtue of [8, Lemma 2.3] there exist an integer $N_{1}(\varepsilon)>0$ and $\delta_{\varepsilon}>0$ such that if $l \geqslant N_{1}(\varepsilon), k \geqslant 2, x_{i} \in C$ $(i=1,2, \cdots, k)$ and if $\left\|x_{i}-x_{j}\right\|-\left\|T^{l} x_{i}-T^{l} x_{j}\right\|<\delta_{\varepsilon}$ for $1 \leqslant i, j \leqslant k$, then $\| T^{l}\left(\sum_{i=1}^{k} r_{i} x_{i}\right)-$ $\sum_{i=1}^{k} r_{i} T^{l} x_{i} \|<\varepsilon$ for every $r=\left(r_{1}, r_{2}, \cdots, r_{k}\right) \in \Delta^{k-1}$, where $\Delta^{k-1}=\left\{r=\left(r_{1}, r_{2}, \cdots, r_{k}\right)\right.$; $r_{i} \geqslant 0(i=1,2, \cdots, k)$ and $\left.\sum_{i=1}^{k} r_{i}=1\right\}$. Consequently, if $l \geqslant N_{1}(\varepsilon), n, m \geqslant 1, x_{i}, y_{i} \in C$ and if $\max \left\{\left\|x_{i}-x_{j}\right\|-\left\|T^{l} x_{i}-T^{l} x_{j}\right\|,\left\|x_{i}-y_{p}\right\|-\left\|T^{l} x_{i}-T^{l} y_{p}\right\|,\left\|y_{p}-y_{q}\right\|-\left\|T^{l} y_{p}-T^{l} y_{q}\right\| ; 0 \leqslant i\right.$, $j \leqslant n-1,0 \leqslant p, q \leqslant m-1\}<\delta_{\varepsilon}$, then

$$
\left\|T^{l}\left(\sum_{i=0}^{n-1} r_{i} x_{i}+\sum_{i=0}^{m-1} t_{i} y_{i}\right)-\left(\sum_{i=0}^{n-1} r_{i} T^{l} x_{i}+\sum_{i=0}^{m-1} t_{i} T^{l} y_{i}\right)\right\|<\varepsilon
$$

for any $r_{i}, t_{i} \geqslant 0$ with $\sum_{i=0}^{n-1} r_{i}+\sum_{i=0}^{m-1} t_{i}=1$. By $(0.3)$ there exists an integer $N_{2}(\varepsilon)>0$ such that $\beta(i)-\delta_{\varepsilon} / 2<\left\|T^{n} x-T^{n+i} x\right\|<\beta(i)+\delta_{\varepsilon} / 2$ for $n \geqslant N_{2}(\varepsilon)$ and $i \geqslant 0$, where $\beta(i)=\lim _{n \rightarrow \infty}\left\|T^{n} x-T^{n+i} x\right\|$. Hence, if $n, m \geqslant N_{2}(\varepsilon)$ and $l \geqslant 0$ then $\left\|T^{i+n} x-T^{j+m} x\right\|-$ $\left\|T^{l+i+n} x-T^{l+j+m} x\right\|<\delta_{\varepsilon} / 2+\beta(|j+m-i-n|)-\left(\beta(|j+m-i-n|)-\delta_{\varepsilon} / 2\right)=\delta_{\varepsilon}$ for $i, j \geqslant 0$. 
So, using (1.7) with $r_{i}=1 / 2 n, x_{i}=T^{i+n} x$ for $0 \leqslant i \leqslant n-1$ and $t_{i}=1 / 2 m, y_{i}=T^{i+m} x$ for $0 \leqslant i \leqslant m-1$, we obtain that if $l, m, n \geqslant \max \left\{N_{1}(\varepsilon), N_{2}(\varepsilon)\right\}$ then $\| T^{l}\left((1 / 2) S_{n} T^{n} x+\right.$ $\left.(1 / 2) S_{m} T^{m} x\right)-(1 / 2)\left(S_{n} T^{l+n} x+S_{m} T^{l+m} x\right) \|<\varepsilon$, i.e., (b) in Theorem 0.1 is satisfied. So, by virtue of Theorem $0.1,\left\{T^{n} x\right\}$ is strongly almost convergent to a fixed point of $T$. Q.E.D.

REMARK 1.2. To prove Theorem 0.2 we have used [8, Lemma 2.3]. As shown in [8], the proof of that lemma is based on [8, Lemma 2.1] which is stated as follows:

Lemma. Suppose that $T$ is asymptotically nonexpansive in the intermediate sense. Then, for $\varepsilon>0$ there exist an integer $N_{\varepsilon}>0$ and $\delta_{2, \varepsilon}>0$ such that if $k \geqslant N_{\varepsilon}, x_{1}, x_{2} \in C$ and if $\left\|x_{1}-x_{2}\right\|-\left\|T^{k} x_{1}-T^{k} x_{2}\right\| \leqslant \delta_{2, \varepsilon}$, then

$$
\left\|T^{k}\left(r_{1} x_{1}+r_{2} x_{2}\right)-r_{1} T^{k} x_{1}-r_{2} T^{k} x_{2}\right\|<\varepsilon
$$

for all $r_{1} \geqslant 0$ and $r_{2} \geqslant 0$ with $r_{1}+r_{2}=1$.

Since the proof in [8] of this lemma is incomplete, we give a proof of the lemma here.

Proof. Let $\delta$ be the modulus of uniform convexity of $X$ and define a function $d:[0, \infty) \rightarrow[0, \infty)$ by

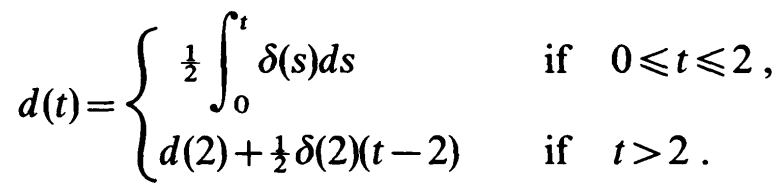

Then $d$ is strictly increasing, continuous and convex, and satisfies

$$
2 r_{1} r_{2} d(\|u-v\|) \leqslant 1-\left\|r_{1} u+r_{2} v\right\|
$$

for $r_{1}, r_{2} \geqslant 0$ with $r_{1}+r_{2}=1,\|u\| \leqslant 1$ and $\|v\| \leqslant 1$.

Let $\varepsilon>0$ be arbitrarily given. Choose an $\eta_{\varepsilon}>0$ such that $\eta_{\varepsilon}<\varepsilon / 3$ and $(D / 4)\left(1+9 D^{2} /\right.$ $\left.\varepsilon^{2}\right) d^{-1}\left(\eta_{\varepsilon} / D\right)<\varepsilon$, and put $\delta_{2, \varepsilon}=\min \left\{\eta_{\varepsilon} / 2, D\right\}$ where $D(>0)$ is the diameter of $C$. By $(0.2)$ there exists an integer $N_{\varepsilon}>0$ such that if $k \geqslant N_{\varepsilon}$ then

$$
\left\|T^{k} p-T^{k} q\right\|<\|p-q\|+\delta_{2, \varepsilon} \quad \text { for every } p, q \in C \text {. }
$$

Let $k \geqslant N_{\varepsilon}$ and let $x_{1}, x_{2} \in C$ with $\left\|x_{1}-x_{2}\right\|-\left\|T^{k} x_{1}-T^{k} x_{2}\right\| \leqslant \delta_{2, \varepsilon}$, and let $r_{1}, r_{2} \geqslant 0$ with $r_{1}+r_{2}=1$.

We first consider the case when $\left\|x_{2}-x_{1}\right\| \geqslant \varepsilon / 3$ and both $r_{1}, r_{2} \geqslant \varepsilon / 3 D$. Put $u=$ $\left(T^{k} x_{2}-T^{k}\left(r_{1} x_{1}+r_{2} x_{2}\right)\right) /\left[r_{1}\left(1+9 D \delta_{2, \varepsilon} / \varepsilon^{2}\right)\left\|x_{2}-x_{1}\right\|\right]$ and $v=\left(T^{k}\left(r_{1} x_{1}+r_{2} x_{2}\right)-T^{k} x_{1}\right) /$ $\left[r_{2}\left(1+9 D \delta_{2, \varepsilon} / \varepsilon^{2}\right)\left\|x_{2}-x_{1}\right\|\right]$. Then $\|u\| \leqslant 1$ and $\|v\| \leqslant 1$ by (1.9), because $\| x_{2}-\left(r_{1} x_{1}+\right.$ $\left.r_{2} x_{2}\right) \geqslant \varepsilon^{2} / 9 D$ and $\left\|r_{1} x_{1}+r_{2} x_{2}-x_{1}\right\| \geqslant \varepsilon^{2} / 9 D$. Since $\left\|r_{1} T^{k} x_{1}+r_{2} T^{k} x_{2}-T^{k}\left(r_{1} x_{1}+r_{2} x_{2}\right)\right\|$ $\leqslant \alpha D\|u-v\| / 2$, where $\alpha=(1 / 2)\left(1+9 D^{2} / \varepsilon^{2}\right)$, by (1.8) and $r_{1} u+r_{2} v=\left(T^{k} x_{2}-T^{k} x_{1}\right) /[(1+$ $\left.\left.9 D \delta_{2, \varepsilon} / \varepsilon^{2}\right)\left\|x_{2}-x_{1}\right\|\right]$ we have 


$$
\begin{aligned}
& d\left((2 / \alpha D)\left\|r_{1} T^{k} x_{1}+r_{2} T^{k} x_{2}-T^{k}\left(r_{1} x_{1}+r_{2} x_{2}\right)\right\|\right) \\
& \quad=d\left((2 / \alpha D) r_{1} r_{2}\left(1+9 D \delta_{2, \varepsilon} / \varepsilon^{2}\right)\left\|x_{2}-x_{1}\right\|\|u-v\|\right) \\
& \quad \leqslant(2 / \alpha D) r_{1} r_{2}\left(1+9 D \delta_{2, \varepsilon} / \varepsilon^{2}\right)\left\|x_{2}-x_{1}\right\| d(\|u-v\|) \\
& \quad \leqslant(1 / \alpha D)\left(\left\|x_{2}-x_{1}\right\|-\left\|T^{k} x_{2}-T^{k} x_{1}\right\|+\left(9 D \delta_{2, \varepsilon} / \varepsilon^{2}\right)\left\|x_{2}-x_{1}\right\|\right) \\
& \quad \leqslant\left(\delta_{2, \varepsilon} / \alpha D\right)\left(1+9 D^{2} / \varepsilon^{2}\right) \leqslant \eta_{\varepsilon} / D .
\end{aligned}
$$

Here we have used the convexity of $d$ and $d(0)=0$. Therefore we obtain from the choice of $\eta_{\varepsilon}$

$$
\left\|r_{1} T^{k} x_{1}+r_{2} T^{k} x_{2}-T^{k}\left(r_{1} x_{1}+r_{2} x_{2}\right)\right\| \leqslant(D / 4)\left(1+9 D^{2} / \varepsilon^{2}\right) d^{-1}\left(\eta_{\varepsilon} / D\right)<\varepsilon .
$$

We next consider the case when $\left\|x_{2}-x_{1}\right\| \geqslant \varepsilon / 3$ and $r_{i}<\varepsilon / 3 D$, where $i=1$ or 2 . By (1.9), $\left\|T^{k}\left(r_{1} x_{1}+r_{2} x_{2}\right)-T^{k} x_{3-i}\right\|<r_{i}\left\|x_{2}-x_{1}\right\|+\delta_{2, \varepsilon}<\varepsilon / 3+\delta_{2, \varepsilon}$ and $\| r_{1} T^{k} x_{1}+r_{2} T^{k} x_{2}-$ $T^{k} x_{3-i}\left\|=r_{i}\right\| T^{k} x_{2}-T^{k} x_{1}\left\|<r_{i}\right\| x_{2}-x_{1} \|+\delta_{2, \varepsilon}<\varepsilon / 3+\delta_{2, \varepsilon}$, which implies

$$
\begin{aligned}
& \left\|T^{k}\left(r_{1} x_{1}+r_{2} x_{2}\right)-\left(r_{1} T^{k} x_{1}+r_{2} T^{k} x_{2}\right)\right\| \\
& \quad \leqslant\left\|T^{k}\left(r_{1} x_{1}+r_{2} x_{2}\right)-T^{k} x_{3-i}\right\|+\left\|T^{k} x_{3-i}-\left(r_{1} T^{k} x_{1}+r_{2} T^{k} x_{2}\right)\right\| \\
& \quad<2 \varepsilon / 3+2 \delta_{2, \varepsilon}<2 \varepsilon / 3+\eta_{\varepsilon}<\varepsilon .
\end{aligned}
$$

Finally, in the case when $\left\|x_{2}-x_{1}\right\|<\varepsilon / 3$ we see from (1.9)

$$
\begin{aligned}
& \left\|T^{k}\left(r_{1} x_{1}+r_{2} x_{2}\right)-\left(r_{1} T^{k} x_{1}+r_{2} T^{k} x_{2}\right)\right\| \\
& \quad \leqslant\left\|T^{k}\left(r_{1} x_{1}+r_{2} x_{2}\right)-T^{k} x_{1}\right\|+\left\|T^{k} x_{1}-\left(r_{1} T^{k} x_{1}+r_{2} T^{k} x_{2}\right)\right\| \\
& \quad<r_{2}\left\|x_{2}-x_{1}\right\|+\delta_{2, \varepsilon}+r_{2}\left\|T^{k} x_{2}-T^{k} x_{1}\right\|<2 r_{2}\left\|x_{2}-x_{1}\right\|+2 \delta_{2, \varepsilon}<2 \varepsilon / 3+\eta_{\varepsilon}<\varepsilon .
\end{aligned}
$$

Q.E.D.

\section{Concluding remarks.}

Definition 2.1 (Bruck [1]). A sequence $\left\{x_{n}\right\}_{n \geqslant 0}$ in $C$ is called an almost-orbit of $T$ if

$$
\lim _{n \rightarrow \infty}\left[\sup _{m \geqslant 0}\left\|x_{n+m}-T^{m} x_{n}\right\|\right]=0 .
$$

The same argument as in $\S 1$ yields the following Theorems 2.1 and 2.2 which are extensions of Theorems 0.1 and 0.2 respectively.

THEOREM 2.1. Let $T$ be asymptotically nonexpansive in the weak sense, and let $\left\{x_{n}\right\}_{n \geqslant 0}$ in $C$ be an almost-orbit of $T$. The following (a) and (b) are equivalent:

(a) $\left\{x_{n}\right\}_{n \geqslant 0}$ is strongly almost convergent to a fixed point of $T$.

(b)

$$
\lim _{l, m, n \rightarrow \infty}\left\|T^{l}\left(\frac{1}{2 n} \sum_{i=0}^{n-1} x_{i+n}+\frac{1}{2 m} \sum_{i=0}^{m-1} x_{i+m}\right)-\left(\frac{1}{2 n} \sum_{i=0}^{n-1} T^{l} x_{i+n}+\frac{1}{2 m} \sum_{i=0}^{m-1} T^{l} x_{i+m}\right)\right\|=0 .
$$


THEOREM 2.2. Let T be asymptotically nonexpansive in the intermediate sense, and let $\left\{x_{n}\right\}_{n} \geqslant 0$ in $C$ be an almost-orbit of $T$. If

$$
\lim _{n \rightarrow \infty}\left\|x_{n+i}-x_{n}\right\| \text { exists uniformly in } i=1,2, \cdots \text {, }
$$

then $\left\{x_{n}\right\}_{n \geqslant 0}$ is strongly almost convergent to a fixed point of $T$.

Acknowledgement. The author is grateful to Professor K. Kobayasi for his valuable comments.

\section{References}

[1] R. E. BRUCK, A simple proof of the mean ergodic theorem for nonlinear contractions in Banach spaces, Israel J. Math. 32 (1979), 107-116.

[2] R. E. BRUCK, T. KuCZumow and S. ReICH, Convergence of iterates of asymptotically nonexpansive mappings in Banach spaces with the uniform Opial property, Colloq. Math. 65 (1993), 169-179.

[3] K. GoEBEL and W. A. KIRK, A fixed point theorem for asymptotically nonexpansive mappings, Proc. Amer. Math. Soc. 35 (1972), 171-174.

[4] W. A. KIRK, Fixed point theorems for non-Lipschitzian mappings of asymptotically nonexpansive type, Israel J. Math. 17 (1974), 339-346.

[5] K. Kobayasi and I. Miyadera. On the strong convergence of the Cèsaro means of contractions in Banach spaces, Proc. Japan Acad. Ser. A 56 (1980), 245-249.

[6] M. KRÜPPEL and J. GóRNICKI, An ergodic theorem for asymptotically nonexpansive mappings, Proc. Roy. Soc. Edinburgh Sect. A 124 (1994), 23-31.

[7] H. OKA, On the nonlinear ergodic theorems for asymptotically nonexpansive mappings in Banach spaces, RIMS (Kyoto Univ.) Kokyuroku 730 (1990), 1-20.

[8] Н. Ока, An ergodic theorem for asymptotically nonexpansive mappings in the intermediate sense, Proc. Amer. Math. Soc. 125 (1997), 1693-1703.

Present Address:

2-5-7 NaKamachi, KoganeI-SHI, ToKyo, 184-0012 JapaN. 